\title{
Dynamo in the Intra-Cluster Medium: Simulation of CGL-MHD Turbulent Dynamo
}

\author{
R. Santos-Lima ${ }^{1}$ E. M. de Gouveia Dal Pino ${ }^{1}$, A. Lazarian ${ }^{2}$, \\ G. Kowal ${ }^{1}$, and D. Falceta-Gonçalves ${ }^{3}$ \\ ${ }^{1}$ IAG, Universidade de São Paulo, Rua do Matão 1226, São Paulo 05508-090, Brazil \\ email: rlima@astro.iag.usp.br \\ 2 Astronomy Department, University of Wisconsin, Madison, WI, USA \\ ${ }^{3}$ NAC, Universidade Cruzeiro do Sul, Rua Galvão Bueno 868, São Paulo 01506-000, Brazil
}

\begin{abstract}
The standard magnetohydrodynamic (MHD) description of the plasma in the hot, magnetized gas of the intra-cluster (ICM) medium is not adequate because it is weakly collisional. In such collisionless magnetized gas, the microscopic velocity distribution of the particles is not isotropic, giving rise to kinetic effects on the dynamical scales. These kinetic effects could be important in understanding the turbulence as well as the amplification and maintenance of the magnetic fields in the ICM. It is possible to formulate fluid models for collisonless or weakly collisional gas by introducing modifications in the MHD equations. These models are often referred as kinetic MHD (KMHD). Using a KMHD model based on the CGL-closure, which allows the adiabatic evolution of the two components of the pressure tensor (the parallel and perpendicular components with respect to the local magnetic field), we performed 3D numerical simulations of forced turbulence in order to study the amplification of an initially weak seed magnetic field. We found that the growth rate of the magnetic energy is comparable to that of the ordinary MHD turbulent dynamo, but the magnetic energy saturates in a level smaller than that of the MHD case. We also found that a necessary condition for the dynamo to operate is to impose constraints on the anisotropy of the pressure.
\end{abstract}

Keywords. magnetic fields, intergalactic medium, plasmas, MHD

\section{Kinetic MHD description of a weakly collisional plasma}

The hypotheses underlying the MHD description of a plasma are not justified when the level of collisionality of the gas is low. Hence, a pure MHD description of the hot, magnetized gas of the ICM medium is not adequate because it is weakly collisional. There, the typical ion Larmour radius $\rho_{i}$ is much smaller that the mean free path $\lambda_{i}$. For instance, in the Hydra A cluster, $\rho_{i} \sim 10^{5} \mathrm{~km}$ and $\lambda_{i} \sim 10^{15} \mathrm{~km}$ (based on data presented in Enßlin \& Vogt 2006).

Nevertheless, it is still possible to formulate fluid models for collisionless or weakly collisional magnetized gas by introducing modifications in the MHD equations. The basic equations of these models are the usual ideal MHD equations for conservation of mass, momentum and the induction equation, with a pressure tensor allowing for distinct parallel and perpendicular components to the magnetic field:

$$
P_{i j}=p_{\perp} \delta_{i j}+\left(p_{\|}-p_{\perp}\right) b_{i} b_{j}
$$

where the $b_{i}$ are the components of the unitary vector parallel to the magnetic field. These models are often referred as kinetic MHD (KMHD). 
The lowest order closure (no heating conduction) to the set of macroscopic equations is given by (Kulsrud 1983):

$$
\frac{d}{d t}\left(\frac{p_{\perp}}{\rho B}\right)=0, \quad \frac{d}{d t}\left(\frac{p_{\|} B^{2}}{\rho^{3}}\right)=0
$$

The resulting model is called CGL-MHD approximation (Chew et al. 1956). These equations of state ensure the conservation of the magnetic momentum of the particles and conservation of entropy of the gas.

The kinetic MHD models reveal linear instabilities originating from the destabilization of the MHD-analogous waves, when the difference between the pressures components reaches some level. The growth rate of these instabilities, in the linear regime, are proportional to the wave number of the perturbation.

We observe the spontaneous development of pressure anisotropy when we perform simulations of forced turbulence using the CGL-MHD model. The instabilities arising from these anisotropies give rise to small-scale structures and therefore, a considerable amount of kinetic and magnetic energy accumulates in the smallest scales of the simulation (limited by the numerical dissipation), changing the usual Kolmogorov power law of the turbulent spectrum in the inertial range. In the absence of numerical dissipation, the CGL-MHD approximation would reveal a serious problem: the growth rate of the highest wavenumbers increases without limits.

\section{Anisotropy limits}

When the magnetic field changes in a frequency higher than the Larmor frequency of the ions, the assumption of conservation of angular momentum is not reasonable anymore, as pitch angle scattering should occur. Therefore, there is a limit to the maximum growth rate of the kinetic instabilities above which the anisotropy of the pressure is reduced as a result of this pitch angle scattering.

Based on kinetic considerations (see Sharma et al. 2006 and references therein), we impose thresholds for the anisotropy of the pressure:

$$
1-\frac{p_{\perp}}{p_{\|}}-\frac{2}{\beta_{\|}} \lesssim \zeta, \quad \frac{p_{\perp}}{p_{\|}}-1 \lesssim \frac{2 \xi}{\beta_{\perp}}
$$

where $\beta_{\|}=p_{\|} /\left(B^{2} / 8 \pi\right)$ and $\beta_{\perp}=p_{\perp} /\left(B^{2} / 8 \pi\right)$. Following Sharma et al. 2006, we use $\zeta=0.5$ and $\xi=3.5$.

When the anisotropy overcomes any of these thresholds, the pitch angle scattering acts to reduce the anisotropy back to the threshold. It imposes some limits to the growth rate of the kinetic instabilities.

\section{Numerical simulation of the CGL-MHD turbulent dynamo}

Using a Godunov-MHD code modified to evolve the CGL-MHD equations (see Kowal et al. 2010), we performed 3D numerical simulations of forced turbulence, starting with a weak seed magnetic field. The turbulence is forced in a periodic box by a random, non-helical, solenoidal force acting around a scale 2.5 times smaller than the side of the cubic computational domain. We work with dimensionless variables defined in terms of the background sound speed and the dimensions of the box. In code units, the random velocity is kept close to unity, hence one turn-over time of the turbulence is $\approx 0.4$. The initial fields are uniform and have the following values: $\rho=1$ (density), $p_{\|}=p_{\perp}=1$, and $B=10^{-4}$ (magnetic field). The resolution employed is $64^{3}$. 


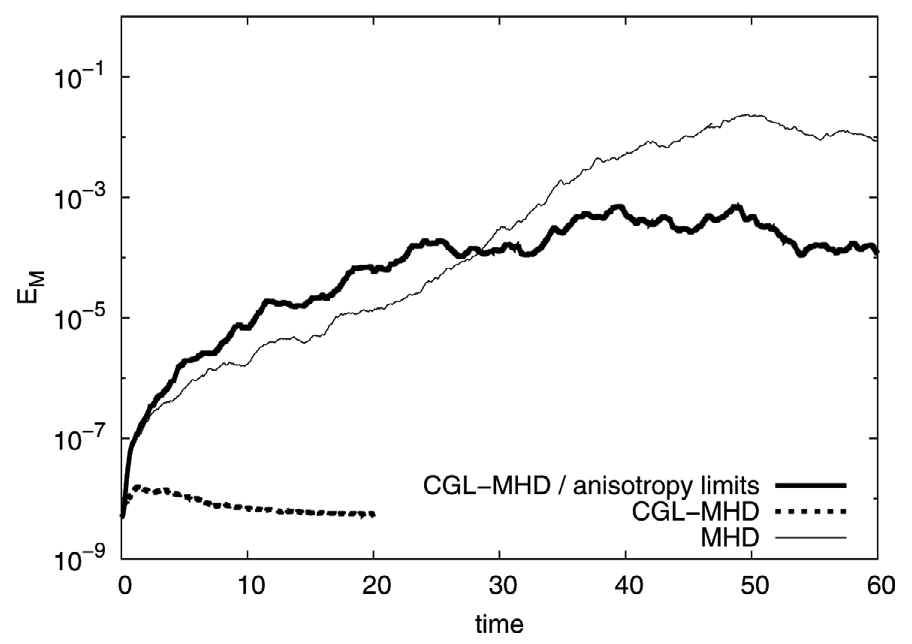

Figure 1. Evolution of the magnetic energy for the CGL-MHD turbulent dynamo. Here we compare two models: with and without the anisotropy limits (see equations 2.1). The ordinary MHD case is showed for comparison. Numerical resolution is $64^{3}$.

Figure 1 shows the evolution of the magnetic energy for two models: one without any constraint on the anisotropy, and another imposing the thresholds of equations (2.1). For comparison, the curve for an ordinary MHD model, employing similar parameters (with an isothermal equation of state) is also shown. For our employed parameters, the growth rate of the magnetic field for the CGL-MHD model with limited anisotropy is similar to the MHD turbulent dynamo. However, the magnetic energy of the CGLMHD model seems to saturate in a value about two orders of magnitude smaller than in the MHD case. For the CGL-MHD model without limited anisotropy, the dynamo fails and the magnetic energy does not grow at all. We also observe failure of the dynamo in simulations employing a KMHD model with isothermal equations of state for the pressure components, whenever $p_{\perp}>p_{\|}$. A suppression of the growth of the magnetic energy by the mirror instability was also detected by Sharma et al. (2006), in the context of magneto-rotational instability.

\section{Conclusions and perspectives}

We have shown that the turbulent CGL-MHD dynamo can amplify the magnetic energy at a rate similar to the MHD turbulent dynamo - provided that we constrain the pressure anisotropy - although the saturation of the magnetic field is much smaller. We still have to assess the influence of the numerical resolution on the results above as well as the influence of the threshold values for the anisotropy. Additionally, the structure of the magnetic field in the saturated state for the CGL-MHD model has to be studied and carefully compared with observations of the ICM.

\section{References}

Chew, G. F., Goldberger, M. L., \& Low, F. E. 1956, Royal Society of London Proceedings Series A, 236, 112

Enßlin, T. A. \& Vogt, C. 2006, A\&A A, 453, 447

Kowal, G., Falceta-Goncalves, D. A., \& Lazarian, A. 2010, arXiv:1012.5125

Kulsrud, R. M. 1983, Basic Plasma Physics: Selected Chapters, Handbook of Plasma Physics, Volume 1, 1

Sharma, P., Hammett, G. W., Quataert, E., \& Stone, J. M. 2006, ApJ, 637, 952 